\title{
An Ionised-impurity Scattering Model for 3D Monte Carlo Device Simulation with Discrete Impurity Distribution
}

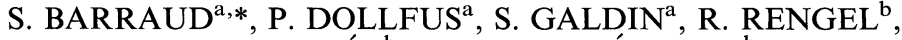 \\ M. J. MARTÍN ${ }^{\mathrm{b}}$ and J. E. VELÁZQUEZ ${ }^{\mathrm{b}}$ \\ a Institut d'Electronique Fondamentale, CNRS UMR 8622, Université Paris-Sud, Bâtiment 220, \\ F-91405 Orsay cedex, France; ${ }^{\mathrm{b}}$ Dpto. De Fisica Aplicada, Universidad de Salamanca, \\ Plaza de la Merced s/n, 37008 Salamanca, Spain
}

\begin{abstract}
An improved 3-D Monte Carlo simulation model is developed to treat the discrete random dopant distribution in sub-0.1 $\mu \mathrm{m}$ MOSFET. The new atomistic model is based on a scattering rate calculation and an algorithm that take into account many-body effects and the local variations of screening length according to impurity distribution and bias conditions.

To validate this new approach low field electron drift mobility and diffusion coefficient have been computed using simulation of 3D bars for $10^{15}-10^{18} \mathrm{~cm}^{-3}$ range of average doping concentration. A good agreement is found between calculation and experimental mobility data at $300 \mathrm{~K}$.
\end{abstract}

Keywords: Doping; Fluctuation; Discrete charge; Monte Carlo; Mobility; Diffusion-coefficient

\section{INTRODUCTION}

To work towards the continuing improvement of performance and density of ULSI technology MOSFET devices are scaled down to sub- $0.1 \mu \mathrm{m}$ dimensions. It results in a decreasing number of channel impurities whose random distribution leads to significant fluctuations of threshold voltage and off-state leakage current [1] which are likely to induce serious consequences on the operation and performance of logical and analog circuits [2]. For such dimensions of active region, impurities cannot be anymore considered as a doping continuum.

In the past years, the effect of discrete random dopant distribution in MOSFET channel has been assessed by analytical [3] or drift-diffusion (DD) [4-6] approaches. The first DD study consisted in using a stochastically fluctuating dopant distribution obeying Poisson statistics [4]. 3-D 'atomistic' simulators $[5,6]$ have been also developed for studying threshold voltage fluctuations.

*Corresponding author. Tel.: +331691540 25, Fax: +331691540 20, e-mail: sylvain.barraud@ief.u-psud.fr 
These 'atomistic' DD approaches consider the discrete impurity distribution while assuming a uniform mobility law. Even though the drift-diffusion method is very useful because of simplicity and fast computing times, 3-D MC simulation should provide a more realistic transport description in ultra-short MOSFET. Indeed, the Monte Carlo procedure provides an exact solution of the Boltzmann transport equation. It thus correctly describes the nonstationary transport conditions and is particularly well suited for noise analysis, since it naturally incorporates all the microscopic noise sources. Wordelman et al., simulate a nMOSFET combining a particle- particle- particlemesh algorithm to calculate the electron-ion interaction with a Monte Carlo method [7].

In this paper, our goal is to propose a proper description of electron transport in the presence of a discrete dopant distribution in the frame of $3 \mathrm{D}$ Monte Carlo simulation. The long-range Coulomb interaction is represented by the 'mesh' electrostatic force resulting from the Poisson equation solution. A local description of the short-range Coulomb interaction is developed by considering the spatial variation of the screened potential according to the charge density and depending on the applied polarisation bias. The many-body contribution is also considered when the local screening length becomes higher than the distance between two ions.

In next section the details of our new approach are described. In Section 3, the electron drift mobility results obtained with both simplified and complete 'atomistic' models are compared with experimental data. The 3-D simulations are carried-out for silicon bars under low electric fields $(0.5 \mathrm{kV} / \mathrm{cm})$. The diffusion coefficients resulting from the analysis of velocity fluctuations are also presented.

\section{THE MODEL}

We use the screened Coulomb interaction to model the carrier-impurity scattering by introducing the momentum-dependent screening length

$$
\beta^{-2}=\frac{e^{2} n(r, t)}{\varepsilon_{0} \varepsilon_{\mathrm{r}} \mathrm{k}_{\mathrm{B}} \mathrm{T}(\mathrm{r}, \mathrm{t})} \mathrm{F}(\xi)
$$

where $\mathrm{k}_{\mathrm{B}}$ is the Boltzmann constant, $\mathrm{n}$ is the local average electron density and $\mathrm{T}$ is the electron temperature. The normalized variable $\xi$ is defined by

$$
\xi^{2}=\frac{\hbar^{2}}{8 \mathrm{em}_{\mathrm{D}} \mathrm{k}_{\mathrm{B}} \mathrm{T}}\left|k^{\prime}-k\right|^{2}
$$

The screening function $\mathrm{F}(\xi)$ defined for nondegenerate semiconductors [8] may be conveniently rewritten as [9]

$$
F(\xi)=\frac{1}{\xi} \exp \left(-\xi^{2}\right) \int_{0}^{\xi} \exp \left(\mathrm{x}^{2}\right) \mathrm{dx}
$$

This is a tabulated function [10] which is approximated for small $\xi$ values by

$$
\mathrm{F}(\xi) \approx\left(1+\frac{2}{3} \xi^{2}\right)^{-1}
$$

Since carrier-impurity scattering is effective only for low energy carrier, we adopt this small- $\xi$ approximation on the whole energy range, which allows to derive analytical expressions of relaxation time. We have chosen indeed to treat this scattering phenomenon as an isotropic mechanism by considering the relaxation time $\tau_{\text {imp }}$ rather than the scattering rate $\lambda_{\text {imp. }}$. This approach has been shown to be equivalent to the corresponding anisotropic model with regard to the resulting computed mobility [11].

\subsection{Carrier-impurity Scattering in Standard MC Simulation}

For large devices, the statistical fluctuations of the impurity distribution have negligible effects on the overall electrical results. Consistently, the classical Monte Carlo method considers impurity atoms as a doping continum characterised by a uniform 
background doping concentration. For simplification, the screening length entering the screened Coulomb potential is calculated for a carrier density equal to the doping concentration. Accordingly, the so-derived relaxation time is only dependent on the doping concentration whatever the local carrier density. In that follows this simple scattering model is referred to as the Standard Model (SM), as summarised in Table I.

\subsection{Carrier-impurity Scattering for Discrete Impurity Distribution}

In the present work, we develop a treatment of carrier-impurity interaction convenient to describe the electron transport in the case of discrete impurity distribution. We have proceeded in two steps to improve the standard model of Coulomb interaction. The first one consists in modifying only the long range "mesh" interaction resulting from the solution of Poisson equation by including impurities as quasi-discrete charges in the device meshing. This simple approach is referred to as the Simplified Atomistic Model (SAM). In the second step, the short range "scattering" mechanism is also modified consistently with a discrete ionised impurity distribution to form the so-called Complete Atomistic Model (CAM).

\subsubsection{Simplified Atomistic Model}

Consider a silicon region of volume $\mathrm{V}$ with a background doping concentration $\mathrm{N}_{\mathrm{I}}$ in which $\mathrm{N}_{\mathrm{at}}=\mathrm{N}_{\mathrm{I}} \times \mathrm{V}$ impurity atoms are randomly distributed. The distribution of the discrete charges is performed by randomly generating the impurity atom positions. Poisson equation is solved by assigning each impurity atom to a cell ' $i$ ' of volume $\mathrm{V}_{\mathrm{i}}$, in which the doping concentration become $\mathrm{N}_{\mathrm{I}}=\mathrm{V}_{\mathrm{i}}{ }^{-1}$. If two or more impurities are assigned to the same cell, the concentration $\mathrm{N}_{\mathrm{I}}$ in this cell is adapted accordingly. This rare case is not considered in expression given in Table I. In a manner similar to the DD atomistic approach mentioned above $[5,6]$, the uniform SM relaxation time is still applied in the whole volume V. Using this simple atomistic model the Monte Carlo procedure and scattering rates are unmodified. Figure 1 illustrates the spatial potential fluctuations induced by a typical discrete impurity distribution in the slice of a silicon bar. The electron density moves accordingly to the potential fluctuations to form a specific electron environment around each impurity.

\subsubsection{Complete Atomistic Model}

In this model the assumption of a single screened potential is given up and the localised screening effects are considered for the relaxation time calculation. This quantity is now locally redefined to take into account the spatial variations of the carrier-density induced by the potential fluctuations. Our approach consists in defining round each impurity a "screening region" in which carriers feel the effect of the screened Coulomb potential (Fig. 2). A scanning of cells surrounding each impurity is thus made to define these regions. The distance $d_{i j}$ between a cell ' $j$ ' (with a zero doping concentration) and the impurity cell ' $i$ ' is compared with the inverse screening length $\beta_{\mathrm{j}}$

TABLE I Description of different models of doping concentration and impurity scattering in the cell 'j' Subscript 'i' corresponds to a cell containing an impurity atom and subscript ' $\mathrm{j}$ ' refers to any cell surrounding this atom

\begin{tabular}{|c|c|c|c|c|c|}
\hline \multirow[b]{2}{*}{$\begin{array}{l}\text { Poisson equation } \\
\nabla^{2} \mathrm{~V}(\mathrm{r})=-\rho_{\mathrm{j}} / \varepsilon_{0} \varepsilon_{\mathrm{r}} \\
\text { Relaxation time } \\
1 /\left(\tau_{\text {imp }}\left(\beta, \mathrm{N}_{1}\right)\right)\end{array}$} & $\begin{array}{l}\text { 1. Standart } \\
\text { Model (SM) }\end{array}$ & 2. Simplified 'Atom & Model (SAM) & \multicolumn{2}{|c|}{ 3. Complete 'Atomistic' Model (CAM) } \\
\hline & $\begin{array}{c}\rho_{\mathrm{j}}=-\mathrm{q}\left(\mathrm{n}_{\mathrm{j}}-\mathrm{N}_{1}\right) \\
\text { whole structure } \\
\qquad \mathrm{N}_{1}=\mathrm{N}_{\mathrm{D}} \\
\beta=\beta\left(\mathrm{n}=\mathrm{N}_{\mathrm{D}}\right)\end{array}$ & $\begin{array}{r}\text { For } j=i \\
\rho_{\mathrm{j}}=-\mathrm{q}\left(\mathrm{n}_{\mathrm{j}}-\mathrm{V}_{1}^{-1}\right) \\
\text { whole str } \\
\mathrm{N}_{1}= \\
\beta=\beta(\mathrm{n}\end{array}$ & $\begin{array}{l}\text { For } j \neq i \\
\rho_{\mathrm{j}}=-\mathrm{qn}_{\mathrm{j}} \\
\text { ure }\end{array}$ & $\begin{array}{c}\text { For } j=i \\
\rho_{\mathrm{j}}=-\mathrm{q}\left(\mathrm{n}_{\mathrm{j}}-\mathrm{V}_{\mathrm{i}}^{-1}\right) \\
\text { Inside of } \mathrm{Vsc} \\
N_{1}=V_{\mathrm{sc}}^{-1} \beta=\beta\left(n=\bar{n}_{\mathrm{sc}}\right)\end{array}$ & $\begin{array}{c}\text { For } j \neq i \\
\rho_{\mathrm{j}}=-\mathrm{qn}_{\mathrm{j}} \\
\text { Outside of } \mathrm{V}_{\mathrm{sc}} \\
1 /\left(\tau_{\mathrm{imp}}\left(\beta, \mathrm{N}_{1}\right)\right)=0\end{array}$ \\
\hline
\end{tabular}




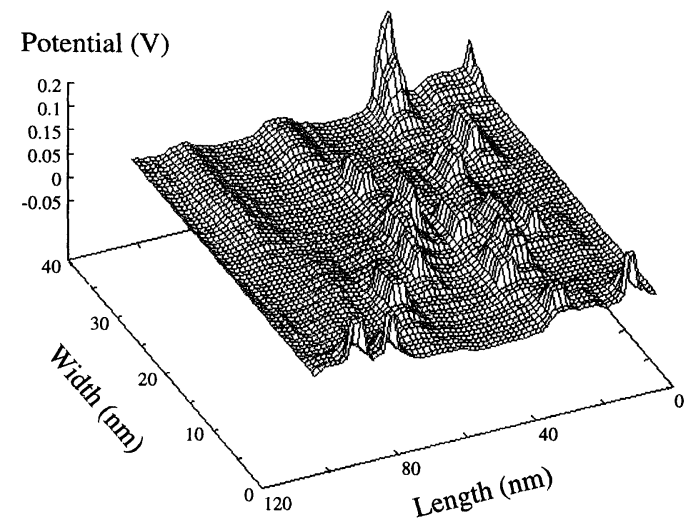

FIGURE 1 Potential fluctuations induced by an "atomistic" doping concentration in a slice of a silicon slab.

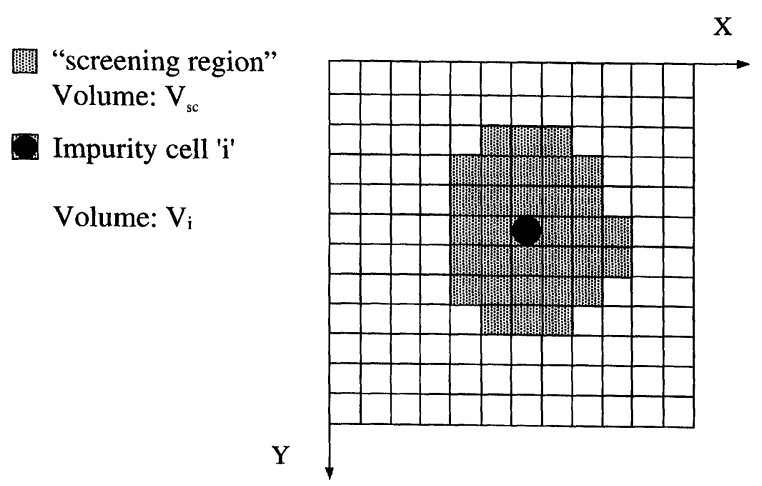

FIGURE 2 Schematised cross section of a "screening region" in a silicon slab (shaded area).

depending on the local carrier density $n_{j}$. The cell ' $\mathrm{j}$ ' is assigned to the screening region only if $\mathrm{d}_{\mathrm{ij}}<\beta_{\mathrm{j}}^{-1}$. In these screening region of volume $\mathrm{V}_{\mathrm{sc}}$, a carrier-impurity scattering rate is applied with an impurity concentration given by $\mathrm{N}_{\mathrm{I}}=\mathrm{V}_{\mathrm{sc}}^{-1}$ and for simplification the inverse screening length considered is the value corresponding to the average density $\mathrm{n}_{\mathrm{sc}}$ in the screening region. Outside of such regions the possibility of carriers-impurity scattering is removed. This algorithm is summarised in Table I.

The relaxation time used above is based on the assumption that an electron may only scatter with a single ion. Many authors pointed out that such approach remains valid only if the average distance between two ions is large compared with the screening length [12]. In MOSFET channel in subthreshold conditions, the low carrier-density yields screening lengths larger than the distance between two scattering centres. Thus, important overlapping zones may appear between the screening regions described above. To include many-body effects in our model the scattering rate is calculated in an overlapping zone by using an ionised-impurity concentration equal to the sum of concentrations of each contributing screening region.

\section{SIMULATION RESULTS- VALIDATION OF THE MODEL}

To check the validity of the atomistic Monte Carlo model described above we first have extracted the low-field electron drift mobility in $\mathrm{Si}$ as a function of the background doping concentration using both SAM and CAM. The conduction band structure used to model the electron transport consists in the classical description of six ellipsoidal $\Delta$ valleys located along the [100] directions at $85 \%$ of the Brillouin zone edge. All details about the band structure and the electron-phonon scattering parameters used in our model may be found in Ref. [13].

The simulated structures are 3-D silicon bars with two terminal contacts and various doping concentrations. The voltage applied to these contacts is fixed and periodic boundary conditions are used, i.e., each carrier leaving the device through a contact is re-injected at the other one with the same wave vector. These scheme allows to maintain a constant number of carriers inside the device. Poisson equation is solved each femtosecond. The impurity atom positions in the bars are randomly chosen. For mobility calculation the bias voltage is chosen so as to induce an average driving field of $0.5 \mathrm{kV} / \mathrm{cm}$. To make significant comparison with experimental bulk mobility, the size of bars is chosen according to the background donor concentration to obtain a donor number 
large enough $\left(\mathrm{N}_{\mathrm{at}}<100\right)$ to recover bulk material transport conditions.

The mobility simulation results are show in Figure 3 together with the experimental data of Ref. [14]. It is noticeable that SAM tends to underestimate the mobility with respect to experimental data. Considering the improved shortrange "scattering" interaction with a screening zone round each impurity atom, a very good agreement is obtained between the CAM and measurements. For comparison, similar results are obtained using the SM in homogeneous transport conditions, which is a supplementary proof that the approach and algorithm used in CAM for discrete impurity distribution is coherent and satisfying.

To evaluate the sensitivity of electron mobility to the size of screening regions, we have artificially doubled and halved the dimensions of these regions for a typical bar with $\mathrm{N}_{\mathrm{D}}=10^{17} \mathrm{~cm}^{-3}$. While the mobility obtain for $\beta=\beta\left(\mathrm{n}_{\mathrm{sc}}\right)$ is 606 $\mathrm{cm}^{2} / \mathrm{Vs}$, in agreement with experimental data, it is $890 \mathrm{~cm}^{2} /$ Vs for $\beta=0.5 \beta\left(\mathrm{n}_{\mathrm{sc}}\right)$ and $280 \mathrm{~cm}^{2} /$ Vs for $\beta=2 \beta\left(\mathrm{n}_{\mathrm{sc}}\right)$. The size of screening regions has thus a strong effect on the mobility and their determination must be made carefully.

Another check of the coherence of the CAM is performed by computing the diffusion coefficient in the same bars from time analysis of velocity

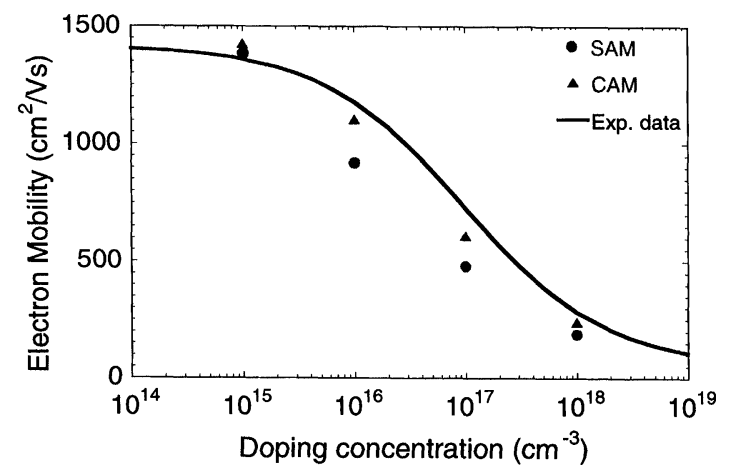

FIGURE 3 Electron mobility as a function of the donor concentration given by the different model: the simplified atomistic model (open circle), the complete atomistic model (triangle). Bars show the experimental mobility data from Masetti et al. [14].

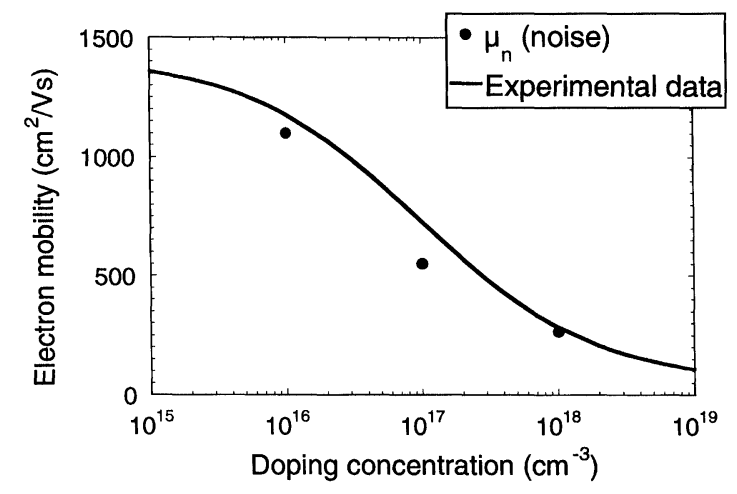

FIGURE 4 Comparison of electron mobility deduced from diffusion coefficient, using the complete atomistic model with the experimental mobility data from Masetti et al. [14].

fluctuations, and more precisely from the spectral density $S_{v}(f)$ of these fluctuations at low frequency [15]. The average electron velocity is recorded each $10 \mathrm{fs}$ over $500 \mathrm{ps}$ to calculate the autocorrelation function of velocity fluctuations $C(t)$ from which is deduced $S_{v}(f)$ [16]. It should be noted that such a computation for $3 \mathrm{D}$ bars requires typically one week of CPU time using up-to-date workstation. In Figure 4 is plotted the mobility deduced from the so-obtained diffusion coefficient through the Einstein relation. A good agreement is still obtained with experimental mobilities.

\section{CONCLUSION}

The standard 3D Monte Carlo algorithm has been modified for studying the effects of the discrete random dopant distribution on electrical parameters of sub- $0.1 \mu \mathrm{m}$ MOSFET. We improve the treatment of Coulomb interactions by including a local screening length according to impurity distribution and carrier density, and by taking into account many-body effects.

The validation of this model is realised from calculation of low field electron mobility and diffusion-coefficient. Comparisons with experimental values show that a simplified atomistic model, modifying only the long range mesh interaction, is not sufficient to obtain a good 
agreement. An appropriate short-range scattering mechanism is necessary to correctly describe electron transport in the case of a discrete ionised impurity distribution. This complete atomistic model is now well suited to study the influence of discrete random dopant distribution on the whole range of MOSFET operation, i.e., from subthreshold to strong inversion regime.

\section{References}

[1] Sitte, R., Dimitrijev, S. and Harrison, H. B. (1994). "Device Parameter Changes Caused by Manufacturing Fluctuations of Deep Submicron MOSFET's", IEEE Trans. Electron Devices, 41, 2210-2215.

[2] Pelgrom, M. J. M. and Vertregt, M. (1997). "CMOS Technology for Mixed Signal IC's”, Solid-state Electron, 41, 967-974.

[3] Stolk, P. A., Widdershoven, F. P. and Klaasen, D. B. M. (1998). "Modeling Statistical Dopant Fluctuations in MOS Transistors", IEEE Trans. Electron Devices, 45, $1960-1971$.

[4] Nishinohara, K., Shigyo, N. and Wada, T. (1992). "Effects of microscopic fluctuations in dopant distributions on MOSFET threshold voltage", IEEE Trans. Electron Devices, 39, 634-639.

[5] Wong, H.-S. and Taur, Y. (1993). "Three-dimensional 'Atomistic' Simulation of Discrete Random Dopant
Distribution Effects in Sub-0.1 $\mu \mathrm{m}$ MOSFET's", In: IEDM Tech. Dig., pp. 705-708.

[6] Asenov, A. (1998). "Random Dopant Induced Threshold Voltage Lowering and Fluctuations in Sub- $0.1 \mu \mathrm{m}$ MOSFET: A 3D “Atomistic' Simulation Study", IEEE Trans. Electron Devices, 45, 2505.

[7] Wordelman, C. J. and Ravaioli, U. (1999). "3-D granular Monte Carlo simulation of silicon n-MOSFETs", Physica $B, 272,568-571$.

[8] Takimoto, N. (1959). "On the screening of impurity potential by conduction electrons", J. Phys. Soc. Jpn., 14, $1142-1158$.

[9] Hall, G. L. (1962). "Ionised Impurity Scattering in Semiconductors", J. Phys. Chem. Solids, 23, 1147-1151.

[10] Abramowitz, M. and Stegun, I. A. (1970). Handbook of Mathematical Functions, New York: Dover publications.

[11] Kosina, H. (1997). "Efficient Evaluation of IonisedImpurity Scattering in Monte Carlo Transport Calculations", Phys. Stat. Sol. (a), 163, 475-489.

[12] Joshi, R. P. and Ferry, D. K. (1991). "Effect of Multi-ion Screening on the Electronic Transport in Doped Semiconductors: A Molecular-Dynamics Analysis", Phys. Rev. $B, 43,9734-9739$.

[13] Dollfus, P. (1997). “ $\mathrm{Si} / \mathrm{Si}_{1-\mathrm{x}} \mathrm{Ge}_{\mathrm{x}}$ heterostructures: electron transport and field-effect transistor operation using Monte Carlo simulation", J. Appl. Phys., 82, 3911-3916.

[14] Masetti, G., Severi, M. and Solmi, S. (1983). IEEE Trans. Electron Devices, 30, 764.

[15] Jacoboni, C. and Lugli, P., The Monte Carlo Method for Semiconductor Device Simulation, Wien: Springer-Verlag, 1989.

[16] Martin, M. J., Valazquez, J. E. and Pardo, D. (1996). "Analysis of current fluctuations in silicon $\mathrm{pn}+$ and $\mathrm{p}+\mathrm{n}$ homojunctions", J. Appl. Phys., 79, 6975-6981. 

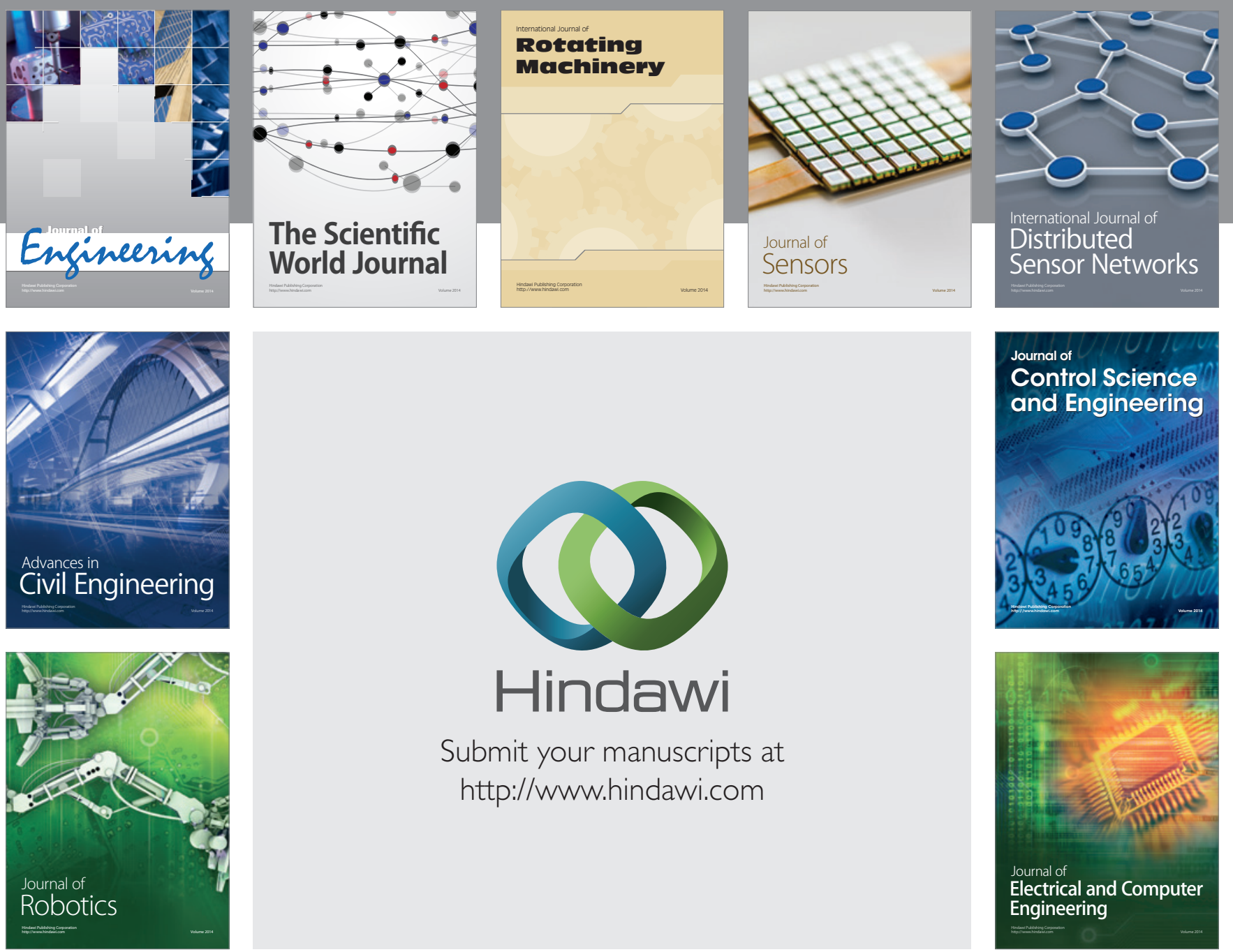

Submit your manuscripts at

http://www.hindawi.com
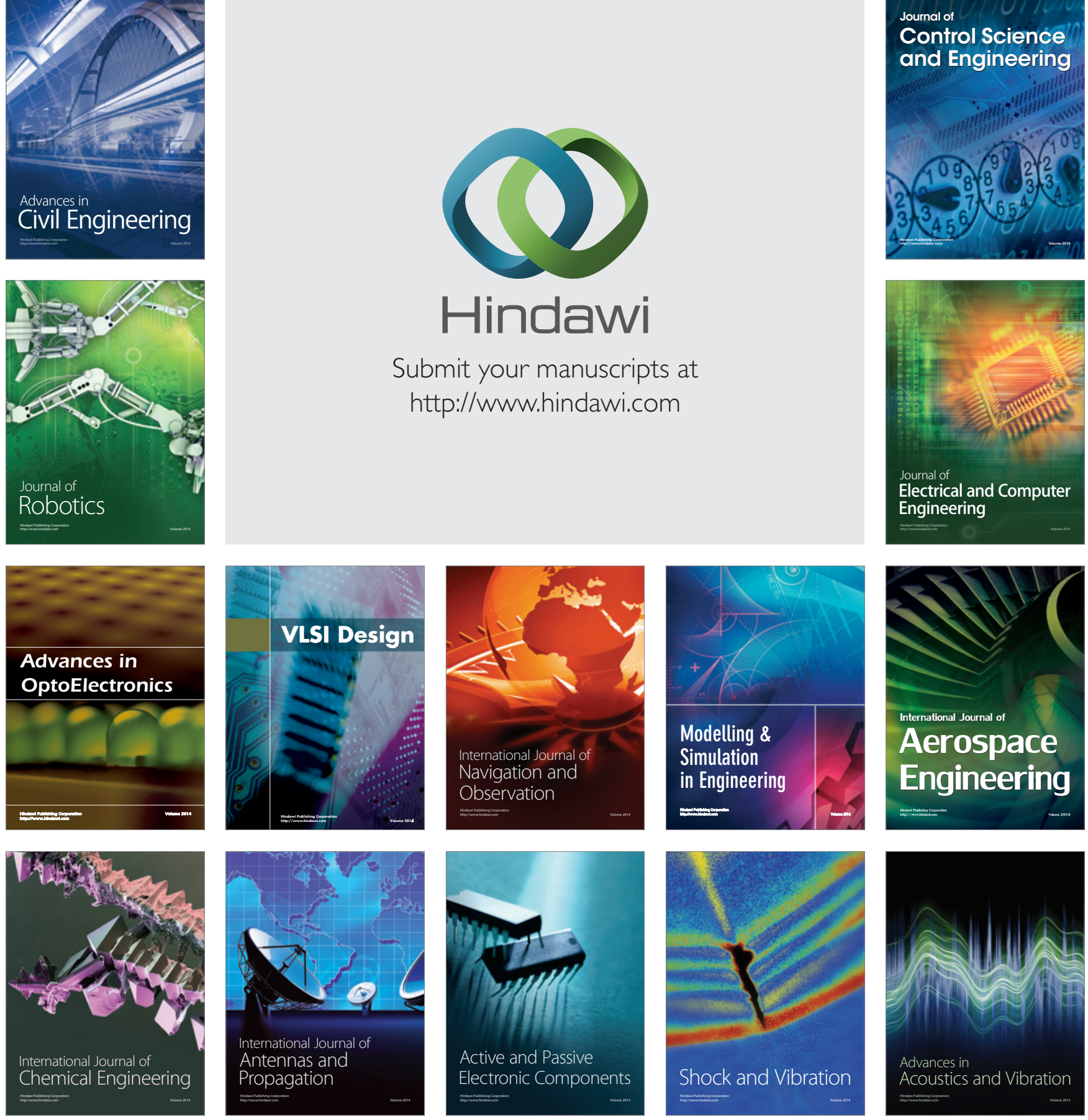